\title{
Sensing Properties of Four-Layered Planar Waveguides - Theoretical Analysis
}

\begin{abstract}
C. TYSZKIEWICZ*
Department of Optoelectronics, Silesian University of Technology, Akademicka 2, 44-100 Gliwice, Poland

Optical waveguides are the fundamental components of chemical and biochemical evanescent wave sensors. Presented work focuses on the theoretical investigations of homogeneous sensitivity of four-layered composite planar waveguide composed of a silica-titania film and a titania film deposited on a glassy substrate in order of citing. Characteristics of a homogeneous sensitivity and a fundamental modes homogeneous sensitivity difference of such a structure are compared with the ones for a three-layered waveguide composed of silica-titania waveguiding film. Therefore the influence of a high refractive index titania film on homogeneous sensitivity characteristics of three-layered structure is given. It was shown that homogeneous sensitivity difference of fundamental modes of four-layered waveguide can be optimized with respect to thicknesses of each layer. For each value of a cover refractive index there exists the minimal thickness of the titania layer which maximizes the homogeneous sensitivity difference of fundamental modes.
\end{abstract}

PACS: 42.79.Gn, 07.07.Df

\section{Introduction}

The continuous and fast progress in scientific research on optical sensors and biosensors is maintained by expanding area for their application. Sensors of this type may find applications in environmental monitoring, food control, pharmacology and in medicine [1]. Application of the optical transducer utilizing evanescent wave spectroscopy enables achieving high sensitivities [2, 3]. The homogeneous sensitivity is a key parameter of planar sensor structures utilizing evanescent wave spectroscopy (planar evanescent wave sensors, PEWS) [4-6]. An optical transducer of a typical PEWS sensor is composed of a slab or a rib waveguide exposed to an ambient of which refractive index changes are detected. Waveguides in use can be either homostructural or heterostructural. Slab and rib waveguides fabricated by means of ion exchange in glass $[7,8]$ fall to the first aforementioned category as well as slab waveguides fabricated by means of sol-gel technology $[9,10]$. Rib waveguides fabricated by means of sol-gel technology are heterostructural. Such waveguides were fabricated by means of selective etching of sol-gel derived $\mathrm{SiO}_{2}-\mathrm{TiO}_{2}$ films and characterized by Karasiński et al. [11-14]. The physical effects behind the operation principle of PEWS may rely on a change of effective indexes of guided modes [15-17]. If the assumption is taken that the refractive index of the homogeneous, semi-infinitive ambient is changed, then the relationship between the small changes of the cover re-

* e-mail: cuma.tyszkiewicz@polsl.pl fractive index and effective refractive index is given by equation

$$
\Delta n_{\mathrm{eff}}=S_{\mathrm{H}} \Delta n_{\mathrm{c}},
$$

where $S_{\mathrm{H}}=\mathrm{d} n_{\text {eff }} / \mathrm{d} n_{\mathrm{c}}$ is the homogeneous sensitivity, $n_{\mathrm{c}}$ is refractive index of the ambient.

The changes of effective indexes can be measured by the application of interferometers. The least technologically complicated is difference interferometer. In single-modal waveguides the fundamental modes TE0 and TM0 are interferencing [4, 18]. Qi et al. suggested the application of a composite waveguide structure along with interference of fundamental modes [19]. Karasiński independently suggested the application of the composite structure composed of uniform silica-titania film/ion-exchange glass optical waveguide in planar differential interferometer configuration with interference of a TM0 and a TM1 mode [20].

This paper is devoted to the theoretical analysis of a relation between the selected geometrical parameters of a composite planar optical waveguide, described in Sect. 2, and its homogeneous sensitivity characteristics. The composite waveguide taken in this paper into consideration is composed of a uniform silica-titania slab waveguide (parent slab) on the top of which a titania film is deposited. Section 3 presents the theoretical analysis of modal, homogeneous sensitivity and fundamental modes homogeneous sensitivity difference characteristics based on characteristic equations.

\section{Composite structure}

The investigated composite planar optical waveguide is composed of a silica-titania slab waveguide (parent 
slab) deposited on a BK7 glass substrate. On a top of this parent slab there is deposited a titania film. The details concerning fabrication technology of the silicatitania and titania films are given in Ref. [11, 21]. The morphological parameters of the investigated composite waveguide as well as of the parent slab waveguide are schematically presented in Fig. 1.

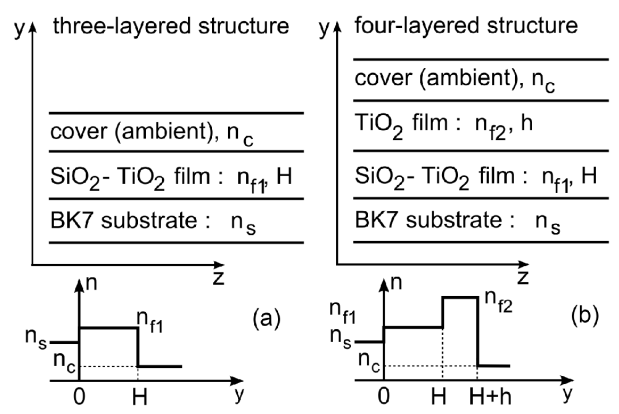

Fig. 1. Diagram of the composite structure: (a) parent slab waveguide, (b) composite waveguide. Parameters description: $n_{f 1}, n_{f 2}, n_{\mathrm{s}}, n_{\mathrm{c}}$ - refractive indexes of a silica-titania film, a titania film, a BK7 substrate, cover (ambient), $H$ - parent slab waveguide thickness, $h-$ thickness of the titania film.

The composite structure is characterized by a thickness $H$ and refractive index $n_{f 1}$ of the silica-titania film, thickness $h$ and refractive index $n_{f 2}$ of the titania film and refractive indexes: $n_{\mathrm{S}}$ of a substrate and $n_{\mathrm{c}}$ of a cover.

The refractive index of silica-titania films has been determined by ellipsometric method using a spectroscopic ellipsometer Woollam M-2000. These studies were carried out in the wavelength range from $190 \mathrm{~nm}$ to $1700 \mathrm{~nm}$. The measurements were performed for three angles of incidence: $60^{\circ}, 65^{\circ}$ and $70^{\circ}$. In case of $\mathrm{SiO}_{2}: \mathrm{TiO}_{2}$ films the spectral dependences of ellipsometer angles $\psi$ and $\Delta$ were fitted with extended Cauchy formula. The dispersion characteristic of the refractive index real part is given by equation

$$
n(\lambda)=A+\frac{B}{\lambda^{2}}+\frac{C}{\lambda^{4}},
$$

where $A=1.751, B=6.077 \times 10^{-3}, C=2.751 \times 10^{-3}$.

The real part of titania refractive index is given by a modified Sellmeier equation [22]. The dispersion characteristic of BK7 glass refractive index is also given by the Sellmeier equation. The detailed information of the Sellmeier coefficients of BK7 were culled from a datasheet published by SCHOOT AG. The spectral dispersion characteristics are shown in Fig. 2. The analysis presented in this paper is carried out for the wavelength $\lambda=677 \mathrm{~nm}$. The values of refractive indexes are as follows: $n_{\mathrm{s}}=1.5137, n_{f 1}=1.7775, n_{f 2}=2.4918$ and $n_{\mathrm{c}}=1.0003$ for dry air and $n_{\mathrm{c}}=1.3310$ for water.

\section{Theoretical analysis}

The analysis of composite waveguide was carried out with the application of characteristic equation method.

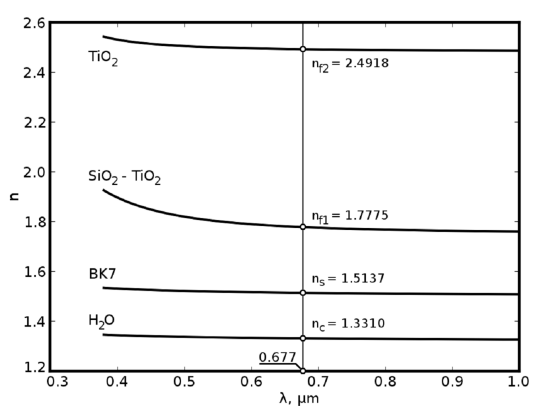

Fig. 2. Refractive index real part chromatic dispersion characteristics $n(\lambda)$ of the silica-titania, titania, BK7 glass and water.

The characteristic equations for parent slab are well known [5] and therefore are not quoted here. In case of the four-layered composite waveguide discussed in this paper there are two characteristic equations depending on an interval an effective index value belongs to. If the effective index fulfils the condition: $n_{f 1}<N<n_{f 2}$, then the characteristic equation is given by Eq. (3). In this case modes are guided in the titania film

$$
\begin{aligned}
& k_{0} h \sqrt{n_{f 2}^{2}-N^{2}}=q \pi+\tan ^{-1}\left(\left(\frac{n_{f 2}}{n_{\mathrm{c}}}\right)^{2 \rho} \sqrt{\frac{N^{2}-n_{\mathrm{c}}^{2}}{n_{f 2}^{2}-N^{2}}}\right) \\
& +\tan ^{-1}\left(\alpha \frac{\beta \cosh (\gamma)+\sinh (\gamma)}{\beta \sinh (\gamma)+\cosh (\gamma)}\right),
\end{aligned}
$$

where

$$
\begin{aligned}
& \alpha=\left(\frac{n_{f 2}}{n_{f 1}}\right)^{2 \rho} \sqrt{\frac{N^{2}-n_{f 1}^{2}}{n_{f 2}^{2}-N^{2}}}, \quad \beta=\left(\frac{n_{f 1}}{n_{\mathrm{s}}}\right)^{2 \rho}, \\
& \gamma=k_{0} H \sqrt{N^{2}-n_{f 1}^{2}},
\end{aligned}
$$

$\rho=0$ for a TE polarization and $\rho=1$ for a TM polarization, $N$ is the effective index of the composite waveguide, $h$ is the thickness of the titania film, $H$ is the thickness of the silica-titania film, $q(=0,1,2, \ldots)$ is the mode number, $n_{f 1}, n_{f 2}, n_{\mathrm{s}}, n_{\mathrm{c}}$ are described in the footnote of Fig. 1. On the other hand, if $n_{\mathrm{s}}<N<n_{f 1}$, then the characteristic equation is given by

$$
\begin{aligned}
k_{0} & \left(H \sqrt{n_{f 1}^{2}-N^{2}}+h \sqrt{n_{f 2}^{2}-N^{2}}\right) \\
& =q \pi+\tan ^{-1}\left(\left(\frac{n_{f 1}}{n_{\mathrm{s}}}\right)^{2 \rho} \sqrt{\frac{N^{2}-n_{\mathrm{s}}^{2}}{n_{f 1}^{2}-N^{2}}}\right) \\
& +\tan ^{-1}\left(\left(\frac{n_{f 2}}{n_{\mathrm{c}}}\right)^{2 \rho} \sqrt{\frac{N^{2}-n_{\mathrm{c}}^{2}}{n_{f 2}^{2}-N^{2}}}\right) \\
& +\tan ^{-1}\left(\chi\left(\frac{n_{f 1}}{n_{f 2}}\right)^{2 \rho} \sqrt{\frac{n_{f 1}^{2}-N^{2}}{n_{f 2}^{2}-N^{2}}}\right)+\tan ^{-1} \chi,
\end{aligned}
$$

where

$$
\chi=\left\{\sin \left(k_{0} h \sqrt{n_{f 2}^{2}-N^{2}}\right)-\left(\frac{n_{f 2}}{n_{\mathrm{s}}}\right)^{2 \rho} \sqrt{\frac{N^{2}-n_{\mathrm{c}}^{2}}{n_{f 2}^{2}-N^{2}}}\right.
$$




$$
\begin{aligned}
& \left.\times \cos \left(k_{0} h \sqrt{n_{f 2}^{2}-N^{2}}\right)\right\} \\
& /\left\{\cos \left(k_{0} h \sqrt{n_{f 2}^{2}-N^{2}}\right)-\left(\frac{n_{f 2}}{n_{\mathrm{s}}}\right)^{2 \rho}\right. \\
& \left.\times \sqrt{\frac{N^{2}-n_{\mathrm{c}}^{2}}{n_{f 2}^{2}-N^{2}}} \sin \left(k_{0} h \sqrt{n_{f 2}^{2}-N^{2}}\right)\right\},
\end{aligned}
$$

where symbols are defined like in the footnote of Eq. (3).

In this case mode turning points are located on an interface between $\mathrm{BK} 7 /$ silica-titania film and an interface between titania film/ambient.

\subsection{Three-layered waveguide modal and homogeneous sensitivity characteristics}

In this paragraph the characteristics of the parent slab waveguide $\left[n_{\mathrm{s}}: n_{f 1}, H: n_{\mathrm{c}}\right]$ are presented. For the three-layered slab the homogeneous sensitivity is given by analytical formulae which can be found in [5] with corrections given in [23]. The spectral characteristic of the parent slab cut-off thickness $H_{\text {cut }}$ are shown in Fig. 3. The values of a cut-off thickness for zero and first order modes are presented in Table I.

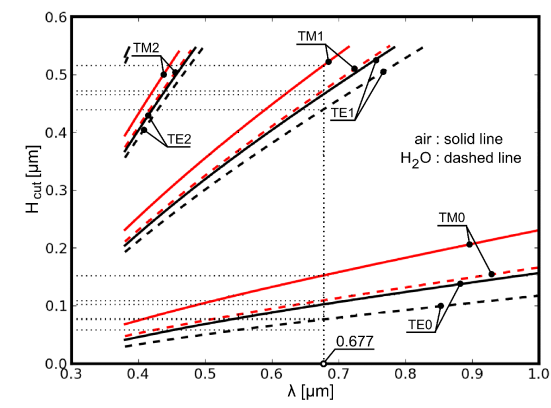

Fig. 3. Cut-off thickness spectral characteristics of the parent. Characteristics calculated for dry air and water as a cover.

\section{TABLE I}

Parent slab cut-off thickness $H_{\text {cut }}[\mu \mathrm{m}]$ for zero and first order TE and TM modes calculated for $\lambda=0.677 \mu \mathrm{m}$.

\begin{tabular}{c|c|c}
\hline \hline & Cover: dry air & Cover: $\mathrm{H}_{2} \mathrm{O}$ \\
\hline TE0 & 0.102 & 0.076 \\
TE1 & 0.465 & 0.439 \\
TM0 & 0.152 & 0.109 \\
TM1 & 0.516 & 0.472
\end{tabular}

The conditions for the single-mode operation regime for parent slab can be read out from Table I. The magnitude of $H_{\text {cut }}$ is decreasing along with increase in $n_{\mathrm{c}}$. In Fig. 4 there are shown the characteristics of the parent slab homogeneous sensitivity $S_{\mathrm{H}}$ in function of a thickness $H$. Maximal $S_{\mathrm{H}}$ values along with corresponding values of thickness $H_{\mathrm{SH} m}$ are presented in Table II.

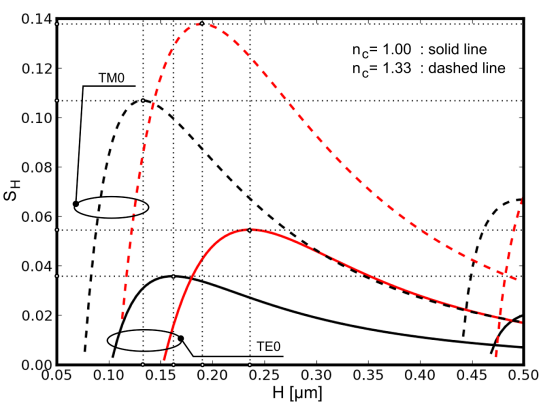

Fig. 4. Homogeneous sensitivity characteristics in function of a thickness of the parent slab. Characteristics calculated for $\lambda=0.677 \mu \mathrm{m}$ and for two values of cover refractive index: $n_{\mathrm{c}}=1.00$ and 1.33 .

TABLE II

Parent slab maximal homogeneous sensitivity $S_{\mathrm{H} m}$ and corresponding thickness $H_{\mathrm{SH} m}[\mu \mathrm{m}]$ for TE0 and TM0 modes calculated for $\lambda=0.677 \mu \mathrm{m}$ and for two values of cover refractive index: $n_{\mathrm{c}}=1.00$ and 1.33 .

\begin{tabular}{c|c|c|c|c}
\hline \hline \multirow{2}{*}{} & \multicolumn{2}{|c|}{$n_{\mathrm{c}}=1.00$} & \multicolumn{2}{c}{$n_{\mathrm{c}}=1.33$} \\
\cline { 2 - 5 } & $S_{\mathrm{H} m}$ & $H_{\mathrm{SH} m}[\mu \mathrm{m}]$ & $S_{\mathrm{H} m}$ & $H_{\mathrm{SH} m}[\mu \mathrm{m}]$ \\
\hline TE0 & 0.036 & 0.162 & 0.107 & 0.132 \\
TM0 & 0.055 & 0.236 & 0.138 & 0.190
\end{tabular}

Maximal sensitivity of a TM mode is greater than of a TE mode and is achieved for greater value of a parent slab thickness. Within the single-mode operation regime only an interference between orthogonally polarised fundamental modes (i.e. TE0-TM0) can be utilized. Therefore a difference between homogeneous sensitivities of these polarisations should be maximized. In Fig. 5 there are shown the characteristics of the parent slab homogeneous sensitivity difference $\Delta S_{\mathrm{H}}$ in function of thickness $H$.

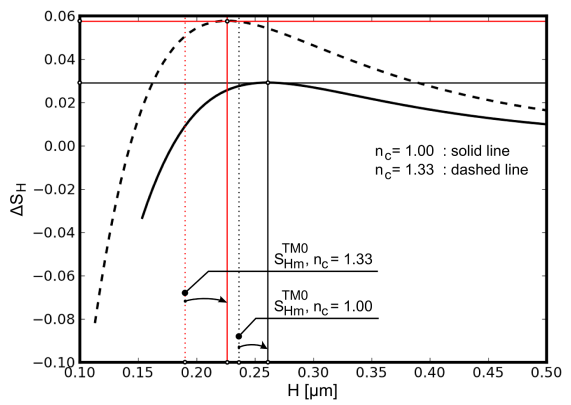

Fig. 5. Homogeneous sensitivity difference characteristics in function of a thickness of the parent slab. Characteristics calculated for $\lambda=0.677 \mu \mathrm{m}$ and for two values of cover refractive index: $n_{\mathrm{c}}=1.00$ and 1.33 .

Dotted lines in Fig. 5 correspond with values of $H_{\mathrm{SH} m}$ for which homogeneous sensitivity of TM0 modes is maximal. It can be seen that the homogeneous sensitivity difference $\Delta S_{\mathrm{H}}$ is maximized for greater thickness values. Maximal $\Delta S_{\mathrm{H}}$ values along with corresponding val- 
ues of thickness $H_{\Delta \mathrm{S} m}$ are presented in Table III. The homogeneous sensitivity difference is increasing with the increase in $n_{\mathrm{c}}$, however these maxima are present for different values of the parent slab thickness. The $H_{\Delta \mathrm{S} m}$ value is decreasing with the increase in $n_{\mathrm{c}}$.

TABLE III

Parent slab maximal homogeneous sensitivity difference $\Delta S_{\mathrm{H} m}$ and corresponding thickness $H_{\Delta \mathrm{S} m}[\mu \mathrm{m}]$ for fundamental modes calculated for $\lambda=0.677 \mu \mathrm{m}$ and for two values of cover refractive index: $n_{\mathrm{c}}=1.00$ and 1.33 .

\begin{tabular}{c|c|c|c|c}
\hline \hline & \multicolumn{2}{|c|}{$n_{\mathrm{c}}=1.00$} & \multicolumn{2}{c}{$n_{\mathrm{c}}=1.33$} \\
\cline { 2 - 5 } & $\Delta S_{\mathrm{H} m}$ & $H_{\Delta \mathrm{S} m}[\mu \mathrm{m}]$ & $\Delta S_{\mathrm{H} m}$ & $H_{\Delta \mathrm{S} m}[\mu \mathrm{m}]$ \\
\hline TE0-TM0 & 0.029 & 0.261 & 0.058 & 0.226
\end{tabular}

\subsection{Four-layered waveguide modal and homogeneous sensitivity characteristics}

In this paragraph we discuss an influence which has an additional high-refractive index titania film that is deposited on the silica-titania parent slab waveguide $\left[n_{\mathrm{s}}: n_{f 1}, H: n_{f 2}, h: n_{\mathrm{c}}\right]$. This structure is schematically presented in Fig. 1b. A presence of the titania film increases the effective indexes of modes because a cover effective index is increased. An effective index of a given mode can exceed the silica-titania refractive index $\left(n_{f 1}\right)$, in which case this mode is confined to the titania film. Following the order of paragraph 3.1, cut-off characteristics for subsequent modes are presented in Fig. 6. The values of $H_{\text {cut }}$ are decreasing along with the increase of the thickness of the titania film. At the beginning of an analysis of a four-layered composite waveguide an assumption is taken that a thickness of a silica-titania film is equal to the value for which the homogeneous sensitivity difference of parent slab is maximized $H_{\Delta \mathrm{S} m}$. From the characteristics presented in Fig. 6 one may read out values of the titania film thickness $h_{\text {cut }}$ for which first order modes are excited. The horizontal dotted lines in Fig. 6 correspond with optimal thicknesses $H_{\Delta \mathrm{S} m}$ of the parent slab. Calculated $h_{\text {cut }}$ values are presented in Table IV.

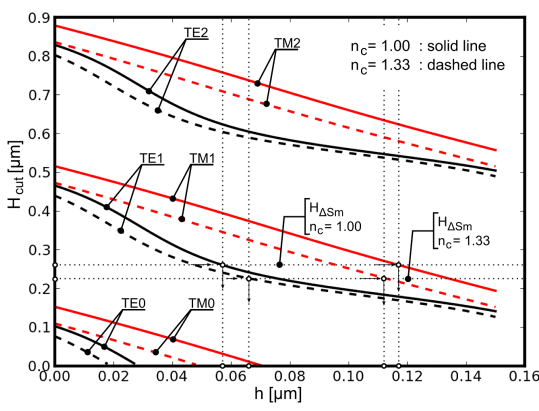

Fig. 6. Cut-off thickness of a silica-titania film in function of a titania film thickness. Characteristics calculated for $\lambda=0.677 \mu \mathrm{m}$ and for two values of cover refractive index: $n_{\mathrm{c}}=1.00$ and 1.33 .

The aforementioned issue regarding a number of layers to which a given mode is confined can be analyzed on

\section{TABLE IV}

Values of a titania film thickness for which first order modes are excited. Calculations are performed for $\lambda=0.677 \mu \mathrm{m}$ and for two values of cover refractive index: $n_{\mathrm{c}}=$ 1.00 and 1.33 .

\begin{tabular}{c|c|c}
\hline \hline \multirow{2}{*}{} & \multicolumn{2}{|c}{$h_{\text {cut }}[\mu \mathrm{m}]$} \\
\cline { 2 - 3 } & TE1 & TM1 \\
\hline$n_{\mathrm{c}}=1.00$ & 0.057 & 0.117 \\
$n_{\mathrm{c}}=1.33$ & 0.066 & 0.112
\end{tabular}

the characteristics of the effective index in function of a titania film thickness. These characteristics for the four-layered composite waveguide $\left[n_{\mathrm{s}}: n_{f 1}, H_{\Delta \mathrm{S} m}: n_{f 2}, h: n_{\mathrm{c}}\right]$ are presented in Fig. 7. For each characteristic one may find a critical thickness $h_{\mathrm{g}}$ for which the following condition is met: $N\left(h_{\mathrm{g}}\right)=n_{f 1}$. If a thickness of the titania film exceeds the $h_{\mathrm{g}}$ then a mode is confined to the titania film. Calculated $h_{\mathrm{g}}$ values for fundamental modes are presented in Table V. From a comparison of values presented in Table IV and Table $\mathrm{V}$ it can be seen that for a given polarization and a mode order $h_{\mathrm{g}}<h_{\text {cut }}$.

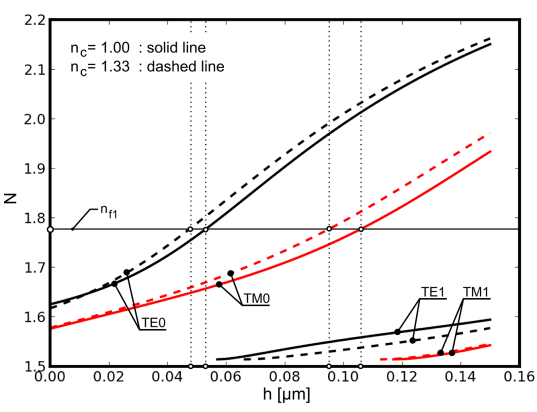

Fig. 7. Effective index characteristics $N(h)$ of the four-layered composite waveguide $\left[n_{\mathrm{s}}: n_{f 1}, H_{\Delta \mathrm{S} m}: n_{f 2}, h: n_{\mathrm{c}}\right]$, whose morphological parameters are presented in Fig. 1b. Characteristics calculated for $\lambda=0.677 \mu \mathrm{m}$ and for two values of cover refractive index: $n_{\mathrm{c}}=1.00$ and 1.33 .

\section{TABLE V}

Values of a critical thickness of a titania film for which first order modes are excited. Calculations are performed for $\lambda=0.677 \mu \mathrm{m}$ and for two values of cover refractive index: $n_{\mathrm{c}}=1.00$ and 1.33 .

\begin{tabular}{c|c|c}
\hline \hline & \multicolumn{2}{|c}{$h_{\mathrm{g}}[\mu \mathrm{m}]$} \\
\cline { 2 - 3 } & TE0 & TM0 \\
\hline$n_{\mathrm{c}}=1.00$ & 0.053 & 0.048 \\
$n_{\mathrm{c}}=1.33$ & 0.106 & 0.095
\end{tabular}

The characteristics of the four-layered waveguide homogeneous sensitivity and homogeneous sensitivity difference in function of $h$ are presented in Fig. 8 and Fig. 9. Maximal $\Delta S_{\mathrm{H}}$ values along with corresponding values of thickness $h_{\Delta \mathrm{S} m}$ are presented in Table VI. 


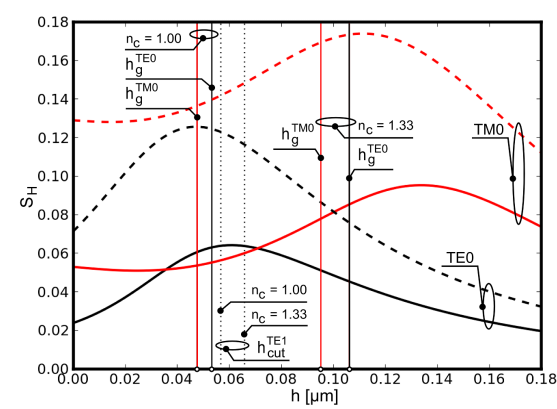

Fig. 8. Homogeneous sensitivity characteristics of the four-layered waveguide in function of a titania film thickness. Characteristics calculated for $\lambda=0.677 \mu \mathrm{m}$ and for two values of cover refractive index: $n_{\mathrm{c}}=1.00$ (solid lines) and 1.33 (dashed lines). The thickness of a silica-titania film is equal to the $H_{\Delta \mathrm{S} m}$ that match given $n_{\mathrm{c}}$.

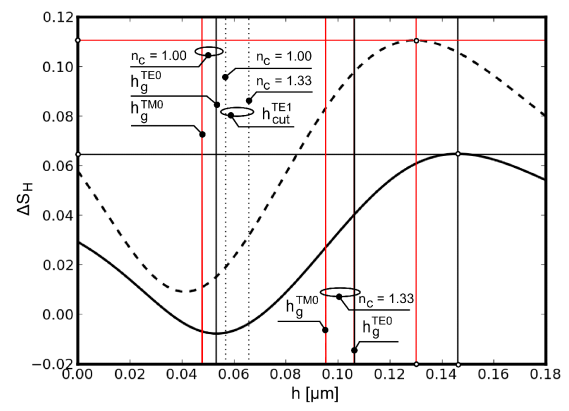

Fig. 9. Homogeneous sensitivity difference characteristics of the four-layered composite waveguide in function of a titania film thickness. Characteristics calculated for $\lambda=0.677 \mu \mathrm{m}$ and for two values of cover refractive index: $n_{\mathrm{c}}=1.00$ (solid lines) and 1.33 (dashed lines). The thickness of a silica-titania film is equal to the $H_{\Delta \mathrm{S} m}$ that match given $n_{\mathrm{c}}$.

It can be seen from Fig. 8 that initial increase in $h$ causes an increase of TE and a decrease of TM modes homogeneous sensitivity. As a result characteristics $\Delta S_{\mathrm{H}}(h)$ have a local minimum before the latter maximum. It is worth mentioning that these maxima are achieved when both TE0 and TM0 modes are confined to the titania film. For each value of a cover refractive index, both TE1 and TM1 modes are present. Therefore the titania film of thickness $h_{\Delta \mathrm{S} m}$ is waveguiding in the

TABLE VI

Four-layered waveguide maximal homogeneous sensitivity difference $\Delta S_{\mathrm{H} m}$ and corresponding thickness $h_{\Delta \mathrm{S} m}[\mu \mathrm{m}]$ for fundamental modes, calculated for $\lambda=$ $0.677 \mu \mathrm{m}$ and for two values of cover refractive index: $n_{\mathrm{c}}=1.00$ and 1.33 .

\begin{tabular}{c|c|c|c|c}
\hline \hline & \multicolumn{2}{|c|}{$n_{\mathrm{c}}=1.00$} & \multicolumn{2}{c}{$n_{\mathrm{c}}=1.33$} \\
\cline { 2 - 5 } & $\Delta S_{\mathrm{H} m}$ & $h_{\Delta \mathrm{S} m}[\mu \mathrm{m}]$ & $\Delta S_{\mathrm{H} m}$ & $h_{\Delta \mathrm{S} m}[\mu \mathrm{m}]$ \\
\hline TE0-TM0 & 0.065 & 0.146 & 0.111 & 0.130
\end{tabular}

discussed four-layered waveguide based on the optimized parent slab, whereas the silica-titania film is not.

As shown above the four-layered waveguide $\left[n_{\mathrm{s}}: n_{f 1}, H_{\Delta \mathrm{S} m}: n_{f 2}, h: n_{\mathrm{c}}\right]$ is poorly optimized. That is because a thickness of the silica-titania film is too high. Further analysis involves a normalized, dimensionless silica-titania film thickness $\eta$ defined with Eq. (6) and a normalized homogeneous sensitivity difference $c$ defined with Eq. (7):

$$
\eta=\frac{H}{H_{\Delta \mathrm{S} m}},
$$

where $H$ is a silica-titania film thickness of a four-layered waveguide, $H_{\Delta \mathrm{S} m}$ is a parent slab thickness which maximizes a homogeneous sensitivity difference of fundamental modes in a three-layered waveguide

$$
c=\frac{\Delta S_{\mathrm{H} m}}{\Delta S_{\mathrm{H} m}^{*}},
$$

where $\Delta S_{\mathrm{H} m}$ is a maximal homogeneous difference of four-layered waveguide, $\Delta S_{\mathrm{H} m}^{*}$ is the parent slab maximal homogeneous sensitivity.

For each pair $\left(\eta, n_{\mathrm{c}}\right)$ the corresponding thickness $h_{\Delta \mathrm{S} m}$ of the titania film maximizing $\Delta S_{\mathrm{H}}$ can be calculated. The characteristics of the optimized thickness $h_{\Delta \mathrm{S} m}$ in function of the normalized thickness $\eta$ are presented in Fig. 10.

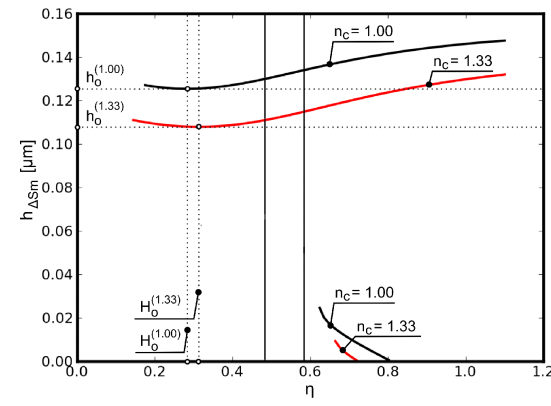

Fig. 10. Characteristics of a titania film thickness $h_{\Delta \mathrm{S} m}$ which maximizes $\Delta S_{\mathrm{H}}$ in function of the normalized thickness $\eta$. Characteristics calculated for $\lambda=$ $0.677 \mu \mathrm{m}$ and for two values of cover refractive index: $n_{\mathrm{c}}=1.00$ and 1.33 .

The characteristics presented in Fig. 10 show that for a given cover refractive index there is a minimal value of the titania film thickness $h_{0}$ for which a homogeneous sensitivity difference is maximized. Moreover, it can be seen that there is a range of normalized parent slab thickness values for which there are two values of $h_{\Delta \mathrm{S} m}$. The span of this range is also dependent on cover refractive index. In Fig. 11 and Fig. 12 there are shown characteristics of a normalized homogeneous sensitivity difference and of an effective index in function of the normalized thickness $\eta$. The cross-section of the horizontal line $N=n_{f 2}$ with TM0 mode effective index characteristics determines a critical normalized thickness $\eta_{\mathrm{g}}$ of silicatitania film. For $\eta>\eta_{\mathrm{g}}$ TM0 modes are confined to the titania film. In Table VII there are presented the cal- 
culated values of $h_{0}$, corresponding values of $H_{0}$, critical values of silica-titania film thickness $H_{\mathrm{g}}$ and values of maximal homogeneous sensitivity difference $\Delta S_{\mathrm{H} m}$.

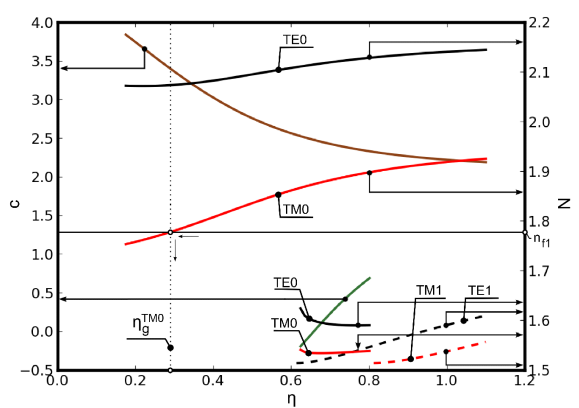

Fig. 11. Effective index and normalized maximal homogeneous sensitivity difference characteristics of the four-layered composite waveguide in function of normalized thickness $\eta$. Characteristics calculated for $\lambda=$ $0.677 \mu \mathrm{m}$ and $n_{\mathrm{c}}=1.00$.

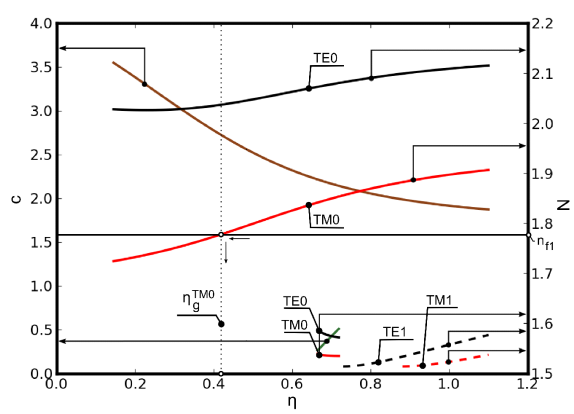

Fig. 12. Effective index and normalized maximal homogeneous sensitivity difference characteristics of the four-layered composite waveguide in function of normalized thickness $\eta$. Characteristics calculated for $\lambda=$ $0.677 \mu \mathrm{m}$ and 1.33 .

TABLE VII

Minimal thickness $h_{0}$ of the titania film maximizing $\Delta S_{\mathrm{H}}$ with associated silica-titania film thickness $\eta_{0} / H_{0}$, critical silica-titania film thickness $\eta_{\mathrm{g}} / H_{\mathrm{g}}^{\mathrm{TMO}}$ and maximal homogeneous sensitivity difference $c_{0} / \Delta S_{\mathrm{H} m}$. Calculations performed for $\lambda=0.677 \mu \mathrm{m}$ and for two values of cover refractive index: $n_{\mathrm{c}}=1.00$ and 1.33 .

\begin{tabular}{c|c|c|c|c}
\hline \hline & $h_{0}[\mu \mathrm{m}]$ & $\eta_{0} / H_{0}[\mu \mathrm{m}]$ & $\eta_{\mathrm{g}} / H_{\mathrm{g}}^{\mathrm{TM}}[\mu \mathrm{m}]$ & $c_{0} / \Delta S_{\mathrm{H} m}$ \\
\hline$n_{\mathrm{c}}=1.00$ & 0.125 & $0.28 / 0.073$ & $0.29 / 0.075$ & $3.42 / 0.100$ \\
$n_{\mathrm{c}}=1.33$ & 0.108 & $0.31 / 0.070$ & $0.42 / 0.094$ & $3.03 / 0.175$
\end{tabular}

From the characteristics presented in Figs. 10, 11 and 12 it can be seen that for the optimal thickness $h_{0}$ of the titania film, where the optimization is in respect of a titania film thickness, the homogeneous sensitivity difference is maximized when $N_{\mathrm{TE} 0}>n_{f 1}$ and $N_{\mathrm{TM} 0}<n_{f 1}$. Comparing maximal $\Delta S_{\mathrm{H}}$ values of the optimized four-layered waveguide with the ones of optimized parent slab waveguide it can be seen that the former waveguide is more sensitive. The magnitude of this increase is dependent on cover refractive index and for $n_{\mathrm{c}}=1.00$ is equal to $c_{0}=3.42$, whereas for $n_{\mathrm{c}}=1.33$ to $c_{0}=3.03$. The characteristics of the four-layered composite waveguide homogeneous sensitivity difference for $H=H_{0}$ in function of $h$ are presented in Fig. 13.

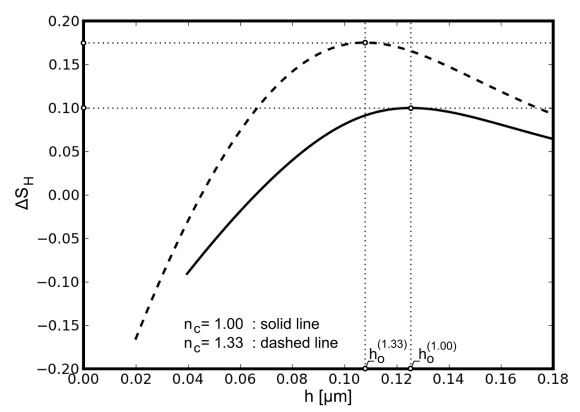

Fig. 13. Homogeneous sensitivity difference characteristics of the four-layered composite waveguide in function of a titania film thickness. Characteristics calculated for $\lambda=0.677 \mu \mathrm{m}$, two values of cover refractive index $\left(n_{\mathrm{c}}=1.00\right.$ and 1.33) and for associated with them $H=H_{0}$.

\section{Conclusions}

This work presents a theoretical analysis of the relation between the geometrical parameters of the four-layered composite silica-titania/titania planar waveguide and its homogeneous sensitivity. The analysis was performed using the method basing on characteristic equations. Effective index and homogeneous sensitivity characteristics of four-layered waveguide are compared with the ones for the optimized three-layered silica-titania waveguide. It was shown that a presence of the titania film on a silica-titania waveguide film, whose thickness $H_{\Delta \mathrm{S} m}$ is optimized in respect of maximization of the three-layered slab $\Delta S_{\mathrm{H}}$, decreases this difference as long as TE0 mode is confined to silica-titania film and TM0 mode effective index is close to or less than a silica-titania film refractive index. For such a structure $\Delta S_{\mathrm{H}}$ is maximal when both fundamental modes are confined to the titania film and first order modes are present. Further analysis of the four-layered composite waveguide of which silica-titania film thickness is less than the aforementioned $H_{\Delta \mathrm{S} m}$, showed that it is possible to find the minimal thickness of the titania film $h_{0}$ which maximizes $\Delta S_{\mathrm{H}}$. For such a structure only TM0 modes are confined to the titania film and fundamental modes are present only. Moreover it was shown that for $h=h_{0}$ there is a range of silica-titania film thickness for which both fundamental modes are guided in both silica-titania and titania film. In this case the maximal homogeneous sensitivity difference is less than for the optimized three-layered parent slab waveguide. 


\section{Acknowledgments}

This work is sponsored by Polish Ministry of Science and Higher Education within a scope of the Grant DEC$-2011 / 01 /$ B /ST7/06525.

\section{References}

[1] G. Boisde, A. Harmer, Chemical and Biochemical Sensing with Optical Fibers and Waveguides, Artech House, Boston 1996.

[2] P. Karasiński, Opto-Electron. Rev. 15, 168 (2007).

[3] P. Karasiński, Opto-Electron. Rev. 19, 10 (2011).

[4] W. Lukosz, Sensors Actuators B 29, 37 (1995).

[5] O. Parriaux, G.J. Veldhuis, J. Lightwave Technol. 16, 573 (1998).

[6] P. Karasiński, C. Tyszkiewicz, R. Rogoziński, Acta Phys. Pol. A 120, 651 (2011).

[7] R. Rogoziński, Opto-Electron. Rev. 13, 229 (2005).

[8] T. Kotyczka, R. Rogoziński, Acta Phys. Pol. A 120, 671 (2011).

[9] P. Karasiński, Proc. SPIE 6608, 66080W (2007).

[10] P. Karasiński, Opt. Appl. 35, 117 (2005).

[11] P. Karasiński, C. Tyszkiewicz, R. Rogoziński, J. Jaglarz, J. Mazur, Thin Solid Films 519, 5544 (2011).
[12] P. Karasiński, P., C. Tyszkiewicz, R. Rogoziński, Opt. Appl. 41, 351 (2011).

[13] P. Karasiński, C. Tyszkiewicz, R. Rogoziński, Photon. Lett. Pol. 2, 40 (2010).

[14] P. Karasiński, R. Rogoziński, Opt. Commun. 245, 237 (2008)

[15] P. Karasiński, Opt. Appl. 32, 775 (2002).

[16] P. Karasiński, R. Rogoziński, Opt. Commun. 281 , 2472 (2008)

[17] C. Tyszkiewicz, Opt. Appl. 42, 555 (2012).

[18] W. Lukosz, Ch. Stamm, H.R. Moser, R. Ryf, J. Dübendorfer, Sensors Actuators B $\mathbf{3 8 / 3 9 ,} 316$ (1997).

[19] Z. Qi, K. Itoh, M. Murabayashi, H. Yanagi, J. Lightwave Technol. 18, 1106 (2000).

[20] P. Karasiński, Opto-Electron. Rev. 19, 1 (2011).

[21] P. Karasiński, J. Sol-Gel Sci. Technol. 61, 355 (2012).

[22] M. Bass, C. DeCusatis, J. Enoch, V. Lakshminarayanan, G. Li, C. MacDonald, V. Mahaja, E. Van Stryland, Handbook of Optics, 3rd ed., Vol. IV, Optical Properties of Materials, Nonlinear Optics, Quantum Optics, McGraw-Hill 2009.

[23] T. El-Agez, S. Taya, Opt. Appl. 41, 89 (2011). 